This is an electronic version of an article published in Spada, Marcantonio M., Gay, Harriet, Nikcevic, Ana, Fernie, Bruce A. and Caselli, Gabriele (2016) Meta-cognitive beliefs about worry and pain catastrophising as mediators between neuroticism and pain behaviour. Clinical Psychologist, 20(3), pp. 138-146.

This can be found online at: http://dx.doi.org/10.1111/cp.12081 


\title{
Metacognitive beliefs about worry and pain catastrophizing as mediators between neuroticism and pain behaviour
}

\author{
Revision 2
}

Regular Article

Word count (all sections included): 5,298

Date of $1^{\text {st }}$ submission: $12 / 06 / 2015$

Date of $2^{\text {nd }}$ submission: $24 / 09 / 2015$

Date of $3^{\text {rd }}$ submission: 08/10/2015

\author{
Marcantonio M. Spada ${ }^{\mathrm{a}, *}$, Harriet Gay ${ }^{\mathrm{a}}$, Ana V. Nikčević ${ }^{\mathrm{b}}$, Bruce A. Fernie ${ }^{\mathrm{c}, \mathrm{d}}$ \\ and Gabriele Caselli ${ }^{\text {a,e }}$
}

${ }^{a}$ Division of Psychology, School of Applied Sciences, London South Bank

University, London, UK

${ }^{\mathrm{b}}$ Department of Psychology, Kingston University, Kingston Upon Thames, UK

${ }^{c}$ King's College London, Institute of Psychiatry, Psychology and Neuroscience, Department of Psychology, London, UK

${ }^{\mathrm{d}}$ CASCAID, South London \&Maudsley NHS Foundation Trust, London, UK

${ }^{\mathrm{e}}$ Studi Cognitivi, Milano, Italy

"Correspondence to: Division of Psychology, School of Applied Sciences, London South Bank University, United Kingdom. Tel. +44 (0)20 7815 5760, e-mail spadam@1sbu.ac.uk.

Acknowledgement

Author BAF receives salary support from the National Institute for Health Research (NIHR) Mental Health Biomedical Research Centre and Dementia Research Unit at South London and Maudsley NHS Foundation Trust and King's College London. KRC is supported in part by NIHR CRN salary. The views expressed are those of the authors and not necessarily those of the NHS, the NIHR, or the Department of Health. 


\begin{abstract}
Objective

The present study explored the relationship between neuroticism, metacognitive beliefs about worry, pain catastrophizing and pain behaviour.
\end{abstract}

\title{
Methods
}

A non-clinical convenience sample of 308 participants completed the following four measures in this cross-sectional study: Neo Five-Factor Inventory, Meta-Cognitions Questionnaire 30, Pain Catastrophizing Scale, and the Pain Behaviour Checklist.

\section{Results}

A multiple-step multiple mediator analysis was employed to test a model in which: (1) positive metacognitive beliefs about worry would mediate the relationship between neuroticism and pain catastrophizing; and (2) negative metacognitive beliefs about worry would mediate the relationship between pain catastrophizing and self-reported pain behaviour. We also hypothesised that the combined effects of metacognitive beliefs about worry and pain catastrophizing on self-reported pain behaviour would be independent of neuroticism. Results supported the proposed structure with pain catastrophizing and metacognitive beliefs about worry mediating fully the effect of neuroticism on self-reported pain behaviour.

\section{Conclusions}

These findings identify, for the first time in the literature, a link between metacognitive beliefs about worry and both self-reported pain behavior and pain catastrophizing. The implications of these findings are discussed.

Key words: cognitive-attentional syndrome; metacognitive beliefs about worry; neuroticism; pain behaviour; pain catastrophizing. 


\section{Key points}

1. We proposed a model of the relationships between neuroticism, metacognitive beliefs about worry, pain catastrophizing and pain behaviour.

2. Results supported the proposed structure with pain catastrophizing and metacognitive beliefs about worry mediating fully the effect of neuroticism on pain behaviour.

3. The present findings suggest that the Self-Regulatory Executive Function model of psychopathology offers a useful framework for developing our understanding of both pain catastrophizing and pain. 


\section{Introduction}

Chronic pain is a common condition that affects up to $19 \%$ of European adults and has deleterious effects on sleep, social functioning, sexual activities, the ability to walk and exercise, and other activities of day-to-day living (Breivik, Collett, Ventafridda, Cohen, \& Gallacher, 2006). It has also a significant impact on mental health, with one study finding that at least one depressive symptom was reported by $16.5 \%$ of individuals with chronic pain (Ohayon \& Schatzberg, 2003) and another contending that pain factors (such as intensity, use of pain medication, and duration of pain) were associated with chronic courses of anxiety and depression over a two year period (Gerrits et al., 2012). Other research has suggested that those with depression comorbid to chronic pain suffer significantly more than those with depression alone (Arnow et al., 2006).

The ways in which people experience and overtly react to pain have been widely examined using observational studies in laboratory and clinical settings (Keefe \& Smith, 2002). Of particular interest to researchers has been the phenomenon of pain catastrophizing, described as the tendency to exaggerate the threat value of pain, as well as a predisposition to respond to it with rumination and worry, which has: (1) been linked to an increase in subjective pain experienced in experimental conditions (Eccleston \& Crombez, 2007; Martel, Trost \& Sullivan, 2012; Sullivan, Rouse, Bishop \& Johnston, 1997) and in chronic pain populations (Sturgeon \& Zautra, 2012); and (2) been shown to influence outward pain behaviours in experimental settings (Sullivan, Rouse, Bishop \& Johnston, 1997; Sullivan, Adams \& Sullivan, 2004). Furthermore, research has suggested that pain catastrophizing or pain-related fear is more disabling (Crombez, Vlaeyen, Heuts, \& Lysens, 1999), and is more emotionally 
distressing (Edwards, Cahalan, Mensing, Smith, \& Haythornthwaite, 2011; Sullivan, Rodgers, \& Kirsch, 2001), than the pain itself.

Neuroticism refers to individual trait differences in negative emotional response to frustration, loss, or threat (Costa \& McCrae, 1992; Goldberg, 1993). Individuals who are high on neuroticism typically respond to day-to-day challenges with emotional responses that are out of proportion to the circumstances (McCrae \& Costa, 2003). In addition, individuals high in neuroticism are often both self-critical and sensitive to the criticism from others, feeling inadequate (Watson, Clark, \& Harkness, 1994). Research findings have linked neuroticism to self-reported pain in patients (Ramírez-Maestre, Martínez \& Zarazaga, 2004), leading to suggestions that neuroticism acts as a 'weak-spot' in pain sufferers, who are then more vulnerable to pain catastrophizing (Goubert, Crombez \& Damme, 2004). In support of this view, individuals high on neuroticism have been observed to be more likely to express medically unfounded somatic complaints (Chaturvedi, 1986; Costa \& McCrae, 1987) as well as report catastrophic thoughts about symptoms that lead to increased healthcare utilisation (Goubert, Crombez, \& Van Damme, 2004).

Metacognition refers to the "stable knowledge or beliefs about one's own cognitive system, and knowledge about factors that affect the functioning of the system; the regulation and awareness of the current state of cognition, and appraisal of the significance of thought and memories" (p.302; Wells, 1995). Research has shown that neuroticism may serve as a 'temperamental basis' for the activation of maladaptive metacognitive responses leading to, and exacerbating, psychological distress (e.g. Dragan et al., 2012; Wells, 2000). In support of this view, metacognitive beliefs (information individuals hold about their own cognition and about coping strategies that impact on inner events) have been found to mediate the relationship 
between neuroticism on the one hand, and dysfunctional behaviour and psychological distress on the other (e.g. Clark et al. 2012, Dragan \& Dragan, 2014; Wells, 2000).

The study of metacognition in psychological disorder and distress is principally associated with the Self-Regulatory Executive Function (S-REF; Wells \& Matthews, 1994; 1996) model. A central tenet of the S-REF model is that metacognitive beliefs play a fundamental role in the persistence of maladaptive forms of coping (e.g. heightened self-focused attention, rumination and worry, and threat monitoring; collectively termed the Cognitive Attentional Syndrome or CAS), which in turn contribute to the development and maintenance of psychological disturbances and distress. According to Wells (2000) metacognitive beliefs can be usefully divided into two broad sets: (1) positive metacognitive beliefs about control strategies that impact on inner events; and (2) negative metacognitive beliefs concerning the significance, controllability, and danger of inner events. In psychological distress the selection and implementation of coping styles based on positive metacognitive beliefs focuses attention towards distress congruent information (e.g. environmental threats). This will typically establish a vicious cycle of faulty CAS blueprints that are consistently applied to alleviate processes appraised as distressing but a successful resolution fails to be achieved. Over time the combination of applying the same CAS blueprint leads to the development of an internal dissonance characterized by negative metacognitive beliefs towards the selected coping styles and internal experiences more generally, leading to the escalation of distress.

The S-REF model is the foundation upon which Metacognitive Therapy (MCT) has been built. MCT aims to alter problematic CAS configurations through interventions designed to modify maladaptive metacognitive beliefs and unhelpful attentional strategies (Wells, 2011). Recently, a meta-analysis suggested that treatment 
outcomes in anxiety disorders and depression achieved by MCT are superior to those resulting from cognitive behaviour therapy (Normann, van Emmerik, \& Morina, 2014). This suggests that if metacognitive targets can be identified, potentially efficacious MCT interventions could be tested in chronic pain samples.

In line with a metacognitive conceptualization, pain catastrophizing may be considered as a coping style similar to rumination and worry and thus a central part of the CAS, with maladaptive consequences including increased levels of pain behaviour. The concept of pain behaviour was originally framed in a behavioural perspective (e.g., Fordyce, 1984) and can be defined as a strategy activated in response to pain which includes overt behaviours that signify an individual is experiencing pain and are contingent on reinforcement schedules as prescribed by operant conditioning. Pain behaviours may consist of avoidance (see Leeuw et al., 2007 for a review), overt means of communication, such as facial displays and vocalizations (Sullivan, Adams, \& Sullivan, 2004), and a heightened vigilance to threatening bodily sensations (Aldrich, Eccleston, \& Crombez, 2000). Such cognitive and attentional processes and behaviours are implicated in problematic CAS configurations. Furthermore, a recent study found that aspects of the CAS (as measured by the Thought Control Questionnaire, Wells \& Davies, 1994), in the form of worry and punishment, were associated with pain catastrophizing and may play a role in maintaining pain-related thoughts and behaviour (Yoshida et al., 2012). Additionally, a study by Turner, Holtzman, and Mancl (2007) found that rumination mediated pain-interference activities as an outcome following cognitive behaviour therapy.

Expanding on Yoshida and colleagues' (2012) work, we propose a model of the relationships between neuroticism, metacognitive beliefs about worry, pain 
catastrophizing, and pain behaviour (presented in Figure 1). In this model neuroticism has an effect on pain catastrophizing through positive metacognitive beliefs about worry (e.g. "Worry can help me cope") that are assumed to play a mediating role by activating pain catastrophizing. Pain catastrophizing, in turn, is associated with pain behaviour through negative metacognitive beliefs about worry (e.g. "I cannot stop worrying"). From an MCT perspective, pain behaviour can be viewed as a maladaptive coping strategy utilised as a form of distraction and/or as a possibly futile attempt to regulate physical and emotional states. If conceptualised as the latter, pain behaviour risks becoming perserverative because it is unlikely to reduce the pain severity or lead to a permanent relief from negative affect. If pain catastrophizing activates negative metacognitive beliefs about worry, then negative affect is likely to increase as a consequence. This may result in the instigation of pain behaviour in an attempt to regulate negative emotional states, thus negative metacognitive beliefs about worry may play a mediating role through the magnification of pain catastrophizing to the extent that pain behaviour ensues.

In summary, using a multiple-step multiple mediator analysis, we tested a model in which: (1) positive metacognitive beliefs about worry would mediate the relationship between neuroticism and pain catastrophizing; and (2) negative metacognitive beliefs about worry would mediate the relationship between pain catastrophizing and pain behaviour. We also hypothesised that the combined effects of metacognitive beliefs about worry and pain catastrophizing on pain behaviour, would be independent of neuroticism. 


\section{Method}

\subsection{Participants and Procedure}

A total of 308 participants (212 females; mean age $=28.9$ years, $\mathrm{SD}=11.8$ years $)$, recruited from a larger student population, completed the study and contributed data used in the analyses. Inclusion criteria were: (1) 18 years of age or above; (2) consenting to the study; (3) understanding spoken and written English; and (4) the absence of any current medical condition which caused chronic or constant pain. A non-clinical sample was chosen for this first exploratory study in order to test the hypothesised relationships before moving on to studies utilising clinical samples. The sample was $82.6 \%$ White, 6.5\% Asian, $1.6 \%$ Black, 6.5\% Mixed, and 2.6\% from another non-specified background.

Ethics approval for the study was obtained from an ethics board of a UK university. A web link directing potential participants to the study website was sent on a university email circular to the student population. A total of 465 individuals took part in the study. Three hundred and nineteen participants met inclusion criteria and completed all the study. Three hundred and eight participants were selected for the final analysis (see Results section). The first page of the study website explained the purpose of the study: "To investigate the relationship between personality, metacognitive beliefs about worry, and pain catastrophizing and behaviour". Participants were then directed, if consenting to participate in the study, to a second page containing basic demographic questions and the self-report instruments. On completion participants were asked to click on the "Submit" button. Once participants had clicked on "Submit", their data was forwarded to a generic postmaster account. This ensured that participants' responses were anonymous. A second submission from 
the same IP address was not allowed so as to avoid multiple submissions from the same participant.

\subsection{Self-report Instruments}

The Neo Five-Factor Inventory (NEO-FFI) (Costa \& McCrae, 1992) is a 60-item self-report instrument assessing personality. It consists of five factors that measure the following dimensions: agreeableness, extraversion, neuroticism, openness and conscientiousness. Higher scores indicate stronger traits. The NEO-FFI has been found to possess excellent psychometric properties (Costa \& McCrae, 1992).

The Meta-Cognitions Questionnaire 30 (MCQ-30; Wells \& CartwrightHatton, 2004) is a 30-item self-report instrument assessing individual differences in metacognitive beliefs, judgments and monitoring tendencies. It consists of five factors that measure the following dimensions: (1) positive beliefs about worry (e.g. "worrying helps me cope"); (2) negative beliefs about thoughts concerning uncontrollability and danger (e.g. "when I start worrying I cannot stop"); (3) cognitive confidence (e.g. "my memory can mislead me at times"); (4) beliefs about the need to control thoughts (e.g. "not being able to control my thoughts is a sign of weakness"); and (5) cognitive self-consciousness (e.g. "I pay close attention to the way my mind works"). Higher scores indicate higher levels of maladaptive metacognitive beliefs. The MCQ-30 possesses good internal consistency and convergent validity, as well as acceptable test-retest reliability (Spada, Mohiyedinni \& Wells, 2008; Wells \& Cartwright-Hatton, 2004).

The Pain Catastrophizing Scale (PCS) (Sullivan, Bishop \& Pivik, 2005) is a 13-item self-report instrument assessing the tendency to catastrophize about pain. It consists of three factors that measure the following dimensions: (1) rumination ("e.g. I can't stop thinking about how much it hurts"); (2) magnification (e.g. "I worry that 
something serious may happen"); and (3) helplessness (e.g. "There is nothing I can do to reduce the intensity of the pain"). Higher total scores indicate increased evidence of pain catastrophizing. The factor structure of the PCS has been replicated in several investigations and shown to possess concurrent validity and good reliability in both clinical and adult non-clinical samples (Osman et al., 1997, 2000; Sullivan, Bishop \& Pivik, 2005; Van Damme et al., 2002).

The Pain Behaviour Checklist (PBC) (Zarkowska, 1981) was used to assess pain behaviour. This 49-item self-report instrument assesses pain-related activities and behaviours (avoidance, complaint, and help-seeking behaviours). The original selfreport instrument obtained categorical data in the form of yes/no answers, but this response format was altered for this study by using a Likert-type scale that ranged from 0-3 ranging (from 'Never' to 'Always'). This was done to broaden the possible range of scores with the goal of increasing the sensitivity of the scale. Higher total scores on the PBC indicate increased evidence of pain behaviour. The original version of the PBC has been validated and shown to be reliable in a sample of individuals suffering headaches (Philips \& Jahanshahi, 1986) but, apparently, not in a non-clinical sample.

\section{Results}

\subsection{Data configuration and descriptive statistics}

An inspection of skewness coefficients showed that all measures were symmetrically distributed. We tested for the presence of multivariate outliers by calculating the distance of Mahalanobis $\left(D^{2}\right)$, which identified eleven participants as multivariate outliers. These were eliminated from further analyses to ensure a linear relationship between variables. The coefficient of Mardia, which represents the multivariate kurtosis coefficient, was 30.3, lower than the critical value (35.0) for an asymmetrical 
multivariate distribution, indicating a multivariate normal distribution. An inspection of graphical distribution of $\mathrm{D}^{2}$ on $\mathrm{Q}-\mathrm{Q}$ plots also supported this finding.

We then examined multicollinearity using the Tolerance Index $\left(T_{i}\right)$ and the Variance Inflation Factor (VIF). A $T_{i}$ over .02 and a value under 5.0 for VIF are considered reliable cut-off points for the absence of multicollinearity. The $T_{i}$ and VIF were measured for neuroticism $\left(T_{i}=.76 ; \mathrm{VIF}=1.31\right)$, pain catastrophizing $\left(T_{i}=.68\right.$; $\mathrm{VIF}=1.48)$, positive beliefs about worry $\left(T_{i}=.89 ; \mathrm{VIF}=1.12\right)$ and negative beliefs about worry $\left(T_{i}=.64 ; \mathrm{VIF}=1.55\right)$. These analyses supported the absence of multicollinearity between variables.

Finally, an inspection of residual Q-Q plots, skewness (-.40), and kurtosis (-.31) showed that: (1) residuals met requirements for normality; (2) there was no indication of non-linearity; and (3) variance was constant for each combination of variables supporting their homoscedasticity. An inspection of correlation coefficients between standardized residuals and independent variables showed that there were no significant correlations. The Durbin-Watson coefficient was 2.06, identifying the absence of autocorrelation. The inspection of Cook's distance and influential data points showed that no participants' data would significantly change the regression analyses coefficients. Descriptive statistics for all variables and Pearson productmoment correlations are presented in Table 1 and showed that all considered variables were positively associated each other. Of note, the Chronbach Alpha for the PBC was excellent and scores significantly and strongly correlated with PCS, providing evidence of the concurrent validity of the former scale in a non-clinical sample.

\subsection{Multiple-step multiple mediator analysis}

To investigate whether the effect of neuroticism could be accounted by metacognitive beliefs and pain catastrophizing we used a multiple-step multiple mediational 
analysis. This test allows verification of the extent to which the proposed chain of mediators carries the influence of an independent variable to a dependent variable. In our model, neuroticism was entered as independent variable, while positive metacognitive beliefs about worry, pain catastrophizing and negative metacognitive beliefs about worry sequentially entered as proposed mediators. Pain behaviour was entered as dependent variable. Statistical analyses were carried out using PROCESS script version 2.13 for SPSS version 21.0 for Windows.

The bootstrap test of indirect effects confirmed that pain catastrophizing and metacognitive beliefs about worry mediated the effect of neuroticism on pain behaviour (See Figure 2). The final equation accounted for $43 \%$ of variance with pain catastrophizing $(t=5.17 ; p<.001, \mathrm{CI}=.59,1.32)$ and negative metacognitive beliefs about worry $(t=2.31 ; p=.02, \mathrm{CI}=.15,1.83)$ as the only significant predictors of pain behaviour. The direct effect of neuroticism on pain behaviour lost its significance $(t=.12 ; p=.91, \mathrm{CI}=-.63, .70)$ when controlling for the interplay between pain catastrophizing and metacognitive beliefs about worry.

The total indirect effect estimate of the whole chain from neuroticism to pain behaviour through positive metacognitive beliefs about worry, pain catastrophizing and negative beliefs about worry in serial was (IE) $=.03,95 \%$ CI $[.02, .04]$. Summary of indirect effects are presented in Table 2.

\section{Discussion}

Overall, the results from this study support the hypothesized model in which: (1) positive metacognitive beliefs about worry would mediate the relationship between neuroticism and pain catastrophizing; and (2) negative metacognitive beliefs about worry would mediate the relationship between pain catastrophizing and self-reported pain behaviour. We also observed, in line with expectations, that the combined effects 
of metacognitive beliefs about worry and pain catastrophizing on self-reported pain behaviour were independent of neuroticism.

These findings identify, for the first time in the literature, a link between metacognitive beliefs about worry and both self-reported pain behavior and pain catastrophizing. According to the metacognitive model of psychopathology metacognitive beliefs are associated with the activation and persistence of particular thinking styles and strategies of mental control (the CAS) in response to negative thoughts and emotions. In our model, positive metacognitive beliefs about worry, which refer to beliefs that worry is a useful coping strategy, may explain the activation of pain catastrophizing as a form of coping in response to an unwanted trigger (e.g. physical sensation, ache, or negative thought). Once pain catastrophizing is initiated, the activation of negative metacognitive beliefs about worry, which refer to the dangers of worry and its uncontrollability, are likely to worsen negative affect. Consequently this may result in an amplification of pain catastrophizing and the engagement in self-reported pain behaviour as an attempt to interrupt distress.

The observed pathways align themselves to the metacognitive model of Generalised Anxiety Disorder (GAD; Wells, 1996) which presents a similar interplay between metacognitive beliefs about worry, worry, and coping strategies employed to regulate escalating worry. The metacognitive model of GAD delineates two types of worry: content-based or Type 1 worry (e.g., 'What if something bad happens?') and meta-worry or Type 2 worry (e.g., 'My worry is uncontrollable'). According to this model, positive metacognitive beliefs about worry initiate Type 1 worry and these activate negative metacognitive beliefs about worry that result in Type 2 worry. Subsequently this exacerbates distress that, because of negative metacognitive beliefs, leads to more worry in an ineffective attempt to regulate emotion, creating a vicious 
cycle. It is possible that pain catastrophizing is exacerbated by an overt form of Type 2 worry, namely self-reported pain behaviour, and that this relationship is mediated by negative metacognitive beliefs about worry.

From a theoretical perspective the present findings suggest that the S-REF model may be helpful in terms of developing an understanding of cognitive, emotional, and behavioural responses to pain, although future research is required to more fully cognize the role of metacognitions. For example, a major limitation of this study is that there could have been time-dependent risk factors that were not considered and these may have had an effect the metacognitive beliefs about worry identified. However, to address this issue, Ecological Momentary Assessment studies could assess whether metacognitive beliefs predict real-time problematic CAS configurations (e.g. engagement in worry during periods of heightened pain experience).

This study has several further limitations that require acknowledgement. Firstly social desirability, self-report biases, context effects, and poor recall may have contributed to errors in self-report measurements. This is to an extent unavoidable as there are no objective or interview measures of metacognitive beliefs. Secondly a cross-sectional design was adopted and this does not allow causal inferences. Thirdly, the study excluded participants with chronic pain conditions; however research has shown that the frequency of pain-related catastrophizing is associated with higher levels of pain intensity and dysfunction when accounting for disease-related variables (e.g. Osborne, Jensen, Ehde, Hanley \& Kraft, 2007; Raichley, Hanley, Jensen \& Cardenas, 2007; Turner, Jensen \& Romano, 2000; Turner, Jensen, Warms \& Cardenas, 2002). Fourthly, this study used the PBC despite the authors being unable to identify other research assessing its psychometric properties in non-clinical 
samples. However, the results suggested that PBC strongly correlate with PCS scores, thus proving some evidence of the concurrent validity of the former in non-clinical samples. Fifthly, the MCQ-30 was used as a measure of metacognitions and was originally developed in a GAD sample. The MCQ-30 measures worry-specific and general metacognitive constructs that may neglect aspects relevant to pain. The use of the Metacognitions about Symptom Control Scale (Fernie, Maher-Edwards, Murphy, Nikcevic, \& Spada, 2015) could yield interesting results in future studies because this measure was designed to assess metacognitive beliefs specific to chronic health conditions: i.e. metacognitive beliefs pertaining to rumination and worry about symptoms, as well as symptom focus. Finally, the similarities between our preliminary findings and the metacognitive model of GAD do not entail that treatment could be transposed without modification, so cautiousness is recommended when interpreting the findings and their possible generalizability to treatment. Despite these limitations, we believe our findings provide preliminary evidence that the S-REF model may be a useful framework to facilitate a broader understanding of the psychological factors associated with pain. 


\section{References}

Aldrich, S., Eccleston, C., \& Crombez, G. (2000). Worrying about chronic pain: vigilance to threat and misdirected problem solving. Behaviour Research and Therapy, 38, 457-470. DOI: 10.1016/S0005-7967(99)00062-5

Arnow, B. A., Hunkeler, E. M., Blasey, C. M., Lee, J., Constantino, M. J., Fireman, B., ... Hayward, C. (2006). Comorbid Depression, Chronic Pain, and Disability in Primary Care. Psychosomatic Medicine, 68, 262-268. DOI: 10.1097/01.psy.0000204851.15499.fc

Breivik, H., Collett, B., Ventafridda, V., Cohen, R., \& Gallacher, D. (2006). Survey of chronic pain in Europe: prevalence, impact on daily life, and treatment. European Journal of Pain, 10, 287-287. DOI: 10.1016/j.ejpain.2005.06.009

Chaturvedi, S. K. (1986). Chronic idiopathic pain disorder. Journal of Psychosomatic Research, 30, 199-203. DOI: 10.1016/0022-3999(86)90050-4

Clark, A., Tran, C., Weiss, A., Caselli, G., Nikčević, A. V. \& Spada, M. M. (2012). Personality and alcohol metacognitions as predictors of levels of alcohol use in binge drinking university students. Addictive Behaviors, 37, 537-540. DOI: 10.1016/j.addbeh.2011.11.035.

Costa, P. T. \& McCrae, R. R. (1987). Neuroticism, somatic complaints and disease: Is the bark worse than the bite. Journal of Personality, 55, 299-316. DOI: 10.1111/j.1467-6494.1987.tb00438.x

Costa, P. T. \& McCrae, R. R. (1992). Revised NEO Personality Inventory (NEO PI-R) and NEO Five-Factor Inventory (NEO-FFI): Professional Manual. Odessa, FL, USA: Psychological Assessment Resources. 
Crombez, G., Vlaeyen, J. W. S., Heuts, P. H. T. G., \& Lysens, R. (1999). Pain-related fear is more disabling than pain itself: evidence on the role of pain-related fear in chronic back pain disability. Pain, 80(1-2), 329-339. DOI: 10.1016/S03043959(98)00229-2

Dragan, M. \& Dragan, W. (2014). Temperament and anxiety: The mediating role of metacognition. Journal of Psychopathological and Behavioural Assessment, 36, 246-254. DOI: 10.1007/s10862-013-9392-z

Dragan, M., Dragan, W., Kononowicz, T. \& Wells A. (2012). On the relationship between temperament, metacognition and anxiety: independent and mediated effects. Anxiety, Stress and Coping, 12, 697-709. DOI: $10.1080 / 10615806.2011 .630071$

Eccleston, C. \& Crombez, G. (2007) Worry and chronic pain: a misdirected problem solving model. Pain, 132, 233-236. DOI: doi:10.1016/j.pain.2007.09.014

Edwards, R. R., Cahalan, C., Mensing, G., Smith, M., \& Haythornthwaite, J. A. (2011). Pain, catastrophizing, and depression in the rheumatic diseases. Nature Reviews Rheumatology, 7, 216-224. DOI: doi: 10.1038/nrrheum.2011.2.

Fernie, B. A., Maher-Edwards, L., Murphy, G., Nikcevic, A. V., \& Spada, M. M. (2015). The Metacognitions about Symptoms Control Scale: Development and Concurrent Validity. Clinical Psychology and Psychotherapy, 22, 443-449. DOI: $10.1002 /$ cpp.1906

Fordyce, W. E. (1984). Behavioural science and chronic pain. Postgraduate Medical Journal, 60, 865-868. DOI:10.1136/pgmj.60.710.865

Gerrits, M. M., Vogelzangs, N., Van Oppen, P., Van Marwijk, H. W., van der Horst, H., \& Penninx, B. W. (2012). Impact of pain on the course of depressive and anxiety disorders. Pain, 153(2), 429-436. DOI: 10.1186/1471-244X-14-187 
Goldberg, L. R. (1993). The structure of phenotypic personality traits. American Psychologist, 48, 26-34. DOI: 10.1037/0003-066X.48.1.26

Goubert, L., Crombez, G. \& Damme, S. (2004). The role of neuroticism, pain catastrophizing and pain-related fear in vigilance to pain: A structural equations approach. Pain, 107, 234-241. DOI: 10.1016/j.pain.2003.11.005

Jensen, M. P., Turner, J. A., Romano, J. M. \& Karoly, P. (1991). Coping with chronic pain: A critical review of the literature. Pain, 47, 249-283. DOI: 10.1016/0304-3959(91)90216-K

Keefe, F. \& Smith, S. (2002). The assessment of pain behavior: Implications for applied psychophysiology and future research directions. Applied Psychophysiology and Biofeedback, 27, 117-127. DOI: 10.1023/A:1016240126437

Leeuw, M., Goossens, M. E., Linton, S. J., Crombez, G., Boersma, K., \& Vlaeyen, J. W. (2007). The fear-avoidance model of musculoskeletal pain: current state of scientific evidence. Journal of Behavioral Medicine, 30, 77-94. DOI: $10.1007 / \mathrm{s} 10865-006-9085-0$

Maher-Edwards, L., Fernie, B. A., Murphy, G., Wells, A. \& Spada, M. M. (2011). Metacognitions and negative emotions as predictors of symptom severity in chronic fatigue syndrome. Journal of Psychosomatic Research, 70, 311-317. DOI: doi:10.1016/j.jpsychores.2010.09.016

Martel, M., Trost, Z. \& Sullivan, M. J. (2012). The expression of pain behaviors in high catastrophizers: The influence of automatic and controlled processes. The Journal of Pain, 13, 808-815. DOI: doi:10.1016/j.jpain.2012.05.015

McCrae, R. R. \& Costa, P. T. (2003). Personality in Adulthood: A Five-factor Theory Perspective. New York, USA: Guilford Press. 
Normann, N., van Emmerik, A. A., \& Morina, N. (2014). The efficacy of metacognitive therapy for anxiety and depression: a meta-analytic review. Depress Anxiety, 31, 402-411. DOI: doi: 10.1002/da.22273

Ohayon, M. M., \& Schatzberg, A. F. (2003). Using chronic pain to predict depressive morbidity in the general population. Archives of General Psychiatry, 60, 3947. DOI: doi:10.1001/archpsyc.60.1.39

Osborne, T. L., Jensen, M. P., Ehde, D. M., Hanley, M. A. \& Kraft, G. (2007). Psychosocial factors associated with pain intensity, pain-related interference, and psychological functioning in persons with multiple sclerosis and pain. Pain, 127, 52-62. DOI: 10.1016/j.apmr.2010.09.021

Osman, A., Barrios, F. X., Kopper, B. A., Hauptmann, W., Jones, J. \& O’Neill, E. (1997). Factor structure, reliability, and validity of the Pain Catastrophizing Scale. Journal of Behavioral Medicine, 20, 589-605. DOI: 10.1023/A:1025570508954

Philips, H. C., \& Jahanshahi, M. (1986). The components of pain behaviour report. Behaviour Research and Therapy, 24, 117-125. DOI: 10.1016/00057967(86)90082-3

Raichle, K. A., Hanley, M., Jensen, M. P. \& Cardenas, D. D. (2007). Cognitions, coping, and social environment predict adjustment to pain in spinal cord injury. Journal of Pain, 8, 718-729. DOI: 10.1016/j.jpain.2007.05.006

Ramírez-Maestre, C., Martínez, A. E. \& Zarazaga, R. E. (2004). Personality characteristics as differential variables of the pain experience. Journal of Behavioral Medicine, 27, 147-165.

DOI: 10.1023/B:JOBM.0000019849.21524.70 
Spada, M. M., Mohiyeddini, C. \& Wells, A. (2008). Measuring metacognitions associated with emotional distress: Factor structure and predictive validity of the Metacognitions Questionnaire 30. Personality and Individual Differences, 45, 238-242. DOI: 10.1016/j.paid.2008.04.005

Sturgeon, J. A. \& Zautra, A. J. (2013). State and trait pain catastrophizing and emotional health in rheumatoid arthritis. Annals of Behavioral Medicine, 45, 69-77. DOI: $10.1007 / \mathrm{s} 12160-012-9408-\mathrm{z}$

Sullivan, M. J. L., Adams, H., \& Sullivan, M. E. (2004). Communicative dimensions of pain catastrophizing: social cueing effects on pain behaviour and coping. Pain, 107, 220-226. DOI: 10.1016/j.pain.2003.11.003

Sullivan, M. J., Bishop, S. R. \& Pivik, J. (1995). The Pain Catastrophizing Scale: Development and validation. Psychological Assessment, 7, 524-532. DOI: $10.1037 / 1040-3590.7 .4 .524$

Sullivan, M. J., Rodgers, W. M., \& Kirsch, I. (2001). Catastrophizing, depression and expectancies for pain and emotional distress. Pain, 91, 147-154. DOI: $10.1016 / \mathrm{S} 0304-3959(00) 00430-9$

Sullivan, M. J., Rouse, D., Bishop, S. \& Johnston, S. (1997). Thought suppression, catastrophizing, and pain. Cognitive Therapy and Research, 21, 555-568. DOI: 10.1023/A:1021809519002

Turner, J. A., Holtzman, S., \& Mancl, L. (2007). Mediators, moderators, and predictors of therapeutic change in cognitive-behavioral therapy for chronic pain. Pain, 127, 276-286. DOI: 10.1016/j.pain.2006.09.005

Turner, J. A., Jensen, M. P. \& Romano, J. M. (2000). Do beliefs, coping, and catastrophizing independently predict functioning in patients with chronic pain? Pain, 85, 115-125. DOI: 10.1016/S0304-3959(99)00259-6 
Turner, J. A., Jensen, M. P., Warms, C. A. \& Cardenas, D. D. (2002). Catastrophizing is associated with pain intensity, psychological distress, and pain-related disability among individuals with chronic pain after spinal cord injury. Pain, 98, 127-134. DOI: 10.1016/S0304-3959(02)00045-3

Van Damme, S., Crombez, G. \& Eccleston, C. (2004). Disengagement from pain: the role of catastrophic thinking about pain. Pain, 107, 70-76. DOI: 10.1016/j.pain.2003.09.023

Wells, A. (1995). Worry and the incubation of intrusive images following stress. Behaviour Research and Therapy, 33, 579-583. DOI: 10.1016/00057967(94)00087-Z

Wells, A. (2000). Emotional Disorders and Metacognition: Innovative Cognitive Therapy. Chichester, UK: Wiley.

Wells, A. (2009). Metacognitive Therapy for Anxiety and Depression. London, UK: Guilford Press.

Wells, A. (2013). Advances in Metacognitive Therapy. International Journal of Cognitive Therapy, 6, 186-201. DOI: 10.1521/ijct.2013.6.2.186

Wells, A. \& Matthews, G. (1994). Attention and Emotion. A Clinical Perspective. Hove, UK: Erlbaum.

Wells, A., \& Cartwright-Hatton, S. (2004). A short form of the metacognitions questionnaire: Properties of the MCQ-30. Behaviour Research and Therapy, 42, 385-396. DOI: 10.1016/S0005-7967(03)00147-5

Wells, A., \& Matthews, G. (1996). Modelling cognition in emotional disorder: The SREF model. Behaviour Research and Therapy, 34(11/12), 881-888. DOI: 10.1016/S0005-7967(96)00050-2 
Yoshida, T., Molton, I. R., Jensen, M. P., Nakamura, T., Arimura, T., Kubo, C. \& Hosoi, M. (2012). Cognitions, metacognitions, and chronic pain. Rehabilitation Psychology, 57, 207-213. DOI: 10.1037/a0030611

Zarkowska, A. W. (1981). The relationship between subjective and behavioral aspects of pain in people suffering from lower back pain (thesis). London, UK: University of London. 
Table 1: Mean, standard deviations (SD), ranges, and two-tailed Pearson correlations of study variables

\begin{tabular}{|c|c|c|c|c|c|c|c|c|}
\hline & Mean & SD & Alpha & Range & 2. & 3. & 4. & 5. \\
\hline $1 . \mathrm{N}$ & 22.8 & 5.4 & .59 & $11-38$ & $.40 * *$ & $.14^{*}$ & $.44 * *$ & $.24 * *$ \\
\hline 2. PCS & 28.24 & 10.6 & .93 & $13-64$ & - & $.28 * *$ & $.52 * *$ & $.43 * *$ \\
\hline 3. PMBW & 10.1 & 3.7 & .87 & $6-24$ & - & - & $.29 * *$ & $.18^{* *}$ \\
\hline 4. NMBW & 11.5 & 4.6 & .90 & $6-24$ & - & - & - & $.33 * *$ \\
\hline 5. PBC & 136.8 & 32.2 & .95 & $49-214$ & - & - & - & - \\
\hline
\end{tabular}

Note. $n=308$; N=Neuroticism; PCS=Pain Catastrophizing Scale; PMBW=Positive Metacognitive Beliefs about Worry; NMBW=Negative Metacognitive Beliefs about Worry; PBC=Pain Behaviour Checklist; *p<.05; ** $p<.01$.

Table 2: Indirect effects and relative confidence intervals

\begin{tabular}{lcrr}
\hline & IE & \multicolumn{2}{c}{ CI } \\
\cline { 2 - 4 } N / PMBW / PBC & & LLCI & ULCI \\
N / PMBW / PCS / PBC & .02 & -.06 & .13 \\
N / PMBW / NMBW / PBC & .06 & .02 & .00 \\
N / PMBW / PCS / NMBW/ PBC & .02 & .02 & .04 \\
N / PCS / PBC & .03 & .42 & .07 \\
N / PCS / NMBW / PBC & .69 & .03 & .23 \\
N / NMBW / PBC & .11 & .04 & .44 \\
\hline
\end{tabular}

Note. $n=308$; N=Neuroticism; PCS=Pain Catastrophizing Scale; PMBW=Positive Metacognitive Beliefs about Worry; NMBW=Negative Metacognitive Beliefs about Worry; PBC=Pain Behaviour Checklist; *p<.05; ** $p<.01$. 
Figure 1: Multiple-step multiple mediational conceptual model of metacognitive beliefs about worry and pain catastrophizing as mediators in serial in the relationship between neuroticism and pain behavior

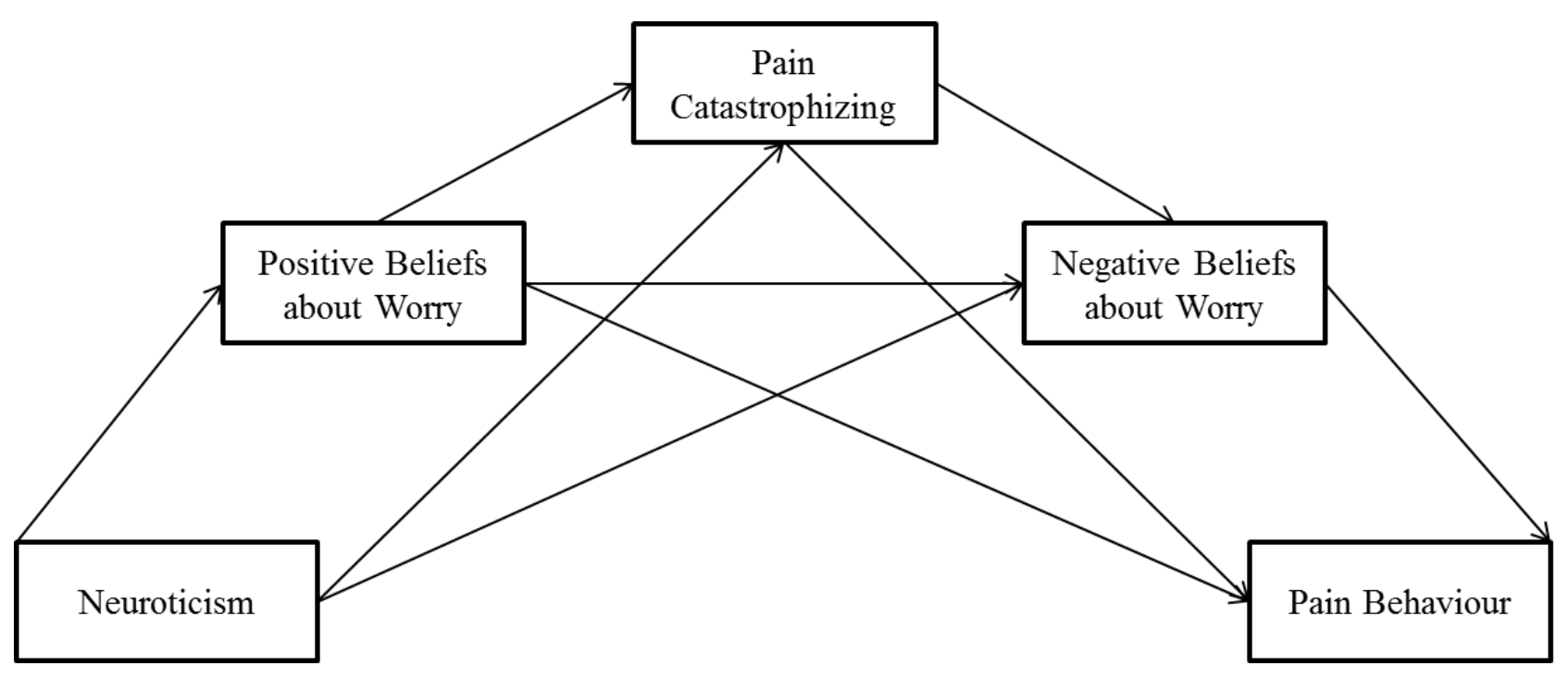


Figure 2: Multiple-step multiple mediational model of metacognitive beliefs about worry and pain catastrophizing as mediators in serial in the relationship between neuroticism and pain behavior $(n=308)$

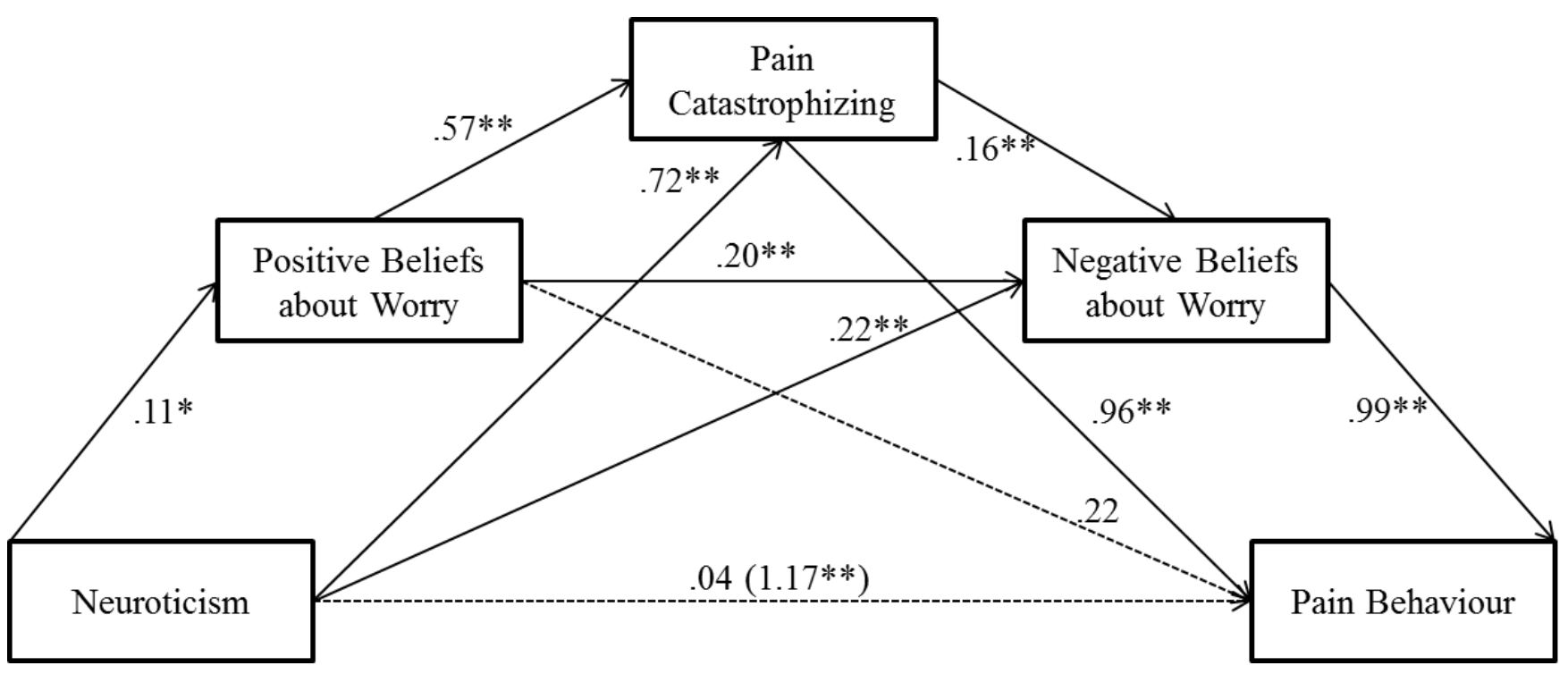

